\title{
ROR2 inhibits the proliferation of gastric carcinoma cells via activation of non-canonical Wnt signaling
}

\author{
LIKUN YAN, QINGGUO DU, JIANFENG YAO and RUITING LIU
}

Department of General Surgery, Shaanxi Provincial People's Hospital, Xi'an, Shaanxi 710068, P.R. China

Received October 26, 2015; Accepted June 23, 2016

DOI: 10.3892/etm.2016.3883

\begin{abstract}
Gastric carcinoma is one of the most common human cancers and has a poor prognosis. Receptor tyrosine kinase-like orphan receptor 2 (ROR2), which is a non-canonical receptor of the Wnt signaling pathway, has been reported to be deregulated in numerous types of human cancers, including gastric carcinoma. However, the exact role of ROR2 in the regulation of the malignant phenotypes of gastric carcinoma, as well as the underlying molecular mechanism, remains largely unclear. The present study demonstrated that ROR2 was recurrently downregulated in gastric carcinoma tissues, as compared with their matched adjacent normal tissues. Furthermore, the expression levels of ROR2 were reduced in several common gastric carcinoma cell lines, as compared with normal gastric epithelial cells. Gastric carcinoma cells were transfected with ROR2 plasmids, and it was demonstrated that restoration of ROR2 expression significantly inhibited the proliferation and induced the apoptosis of gastric carcinoma cells by a Wnt5a-independent mechanism. In addition, it was observed that ROR2-overexpressing cells accumulated in the G0/G1 phase; thus suggesting that overexpression of ROR2 induced cell cycle arrest at the G0/G1 phase. An investigation of the underlying mechanism demonstrated that activation of the non-canonical Wnt signaling pathway inhibited canonical Wnt signal transduction, as demonstrated by the decreased level of $\beta$-catenin in nuclei, as well as the reduced expression levels of c-Myc. The results of the present study indicated a tumor suppressive role for ROR2 in gastric carcinoma growth in vitro, and suggested that ROR2 may be used as a molecular target for the treatment of gastric carcinoma.
\end{abstract}

\section{Introduction}

Gastric carcinoma is one of the most common human cancers and has a high mortality rate (1). Although significant progress

Correspondence to: Dr Likun Yan, Department of General Surgery, Shaanxi Provincial People's Hospital, 256 Youyi Xi Road, Xi'an, Shaanxi 710068, P.R. China

E-mail: shanxiyanlikun@163.com

Key words: gastric carcinoma, receptor tyrosine kinase-like orphan receptor 2, proliferation, Wnt signaling in the treatment of gastric carcinoma (involving surgical excision used in combination with chemotherapy) has been achieved in recent years, the median survival rate of gastric carcinoma remains low (2). The downregulation of tumor suppressor genes has been implicated in the tumorigenesis of gastric carcinoma $(3,4)$. Therefore, elucidation of the molecular mechanisms underlying gastric carcinoma is important for the development of therapeutic strategies for this disease.

Receptor tyrosine kinase-like orphan receptor 2 (ROR2) is a member of the receptor tyrosine kinase family (5). It has been demonstrated that ROR2 is involved in numerous cellular biological processes, including cell proliferation, differentiation, apoptosis, adhesion and migration (6-8). Furthermore, ROR2 is a receptor of the non-canonical Wnt signaling pathway, and Wnt5a has been determined to be the most important ligand of ROR2 (9). In a previous study, the expression levels of ROR2 were observed to be recurrently downregulated in various common human cancers, including esophageal, nasopharyngeal, colorectal, gastric, hepatocellular, lung and breast cancer (10). Accordingly, it has been suggested that ROR2 may act as a tumor suppressor in human cancers. However, ROR 2 has been reported to have an oncogenic role in other types of cancer $(11,12)$. For instance, ROR2 was shown to be upregulated in osteosarcoma tissues, and its expression level was positively correlated with disease severity (11). In addition, ROR2 was reported to increase the tumor growth potential in renal cell carcinoma (RCC) (12). These findings suggest that ROR2 may have dual roles in various cancer types. However, to the best of our knowledge, the exact role of ROR2 in the regulation of gastric carcinoma growth, as well as the underlying mechanism, have yet to be investigated.

The present study aimed to investigate the role of ROR2 in the regulation of the proliferation, apoptosis and cell cycle progression of gastric carcinoma cells, as well as the underlying molecular mechanisms.

\section{Materials and methods}

Tissue specimen collection. All protocols involved in the present study were approved by the Ethics Committee of Shaanxi Provincial People's Hospital (Xi'an, China). A total of 20 gastric carcinoma tissue samples and their matched adjacent normal tissue samples were collected from patients at the Shaanxi Provincial People's Hospital between June 2013 and January 2014. Of the 20 patients, 13 were male and 7 were female. The patients had a mean age of 53.7 years (age 
range, 33-77 years). The patients did not receive radiation therapy or chemotherapy prior to surgical resection. Informed consent was obtained from all patients. The resected tissue samples were immediately snap-frozen in liquid nitrogen and stored at $-70^{\circ} \mathrm{C}$ prior to use.

Cell culture. Four common human gastric cancer cell lines (BGC823, SGC7901, HGC27 and AGS), and a normal gastric mucosa epithelial cell line (GES-1), were obtained from the Type Culture Collection of the Chinese Academy of Sciences (Shanghai, China). All cell lines were cultured in Dulbecco's modified Eagle's medium(DMEM) supplemented with $10 \%$ fetal bovine serum (both purchased from Thermo Fisher Scientific, Inc., Waltham, MA, USA) in a humidified incubator containing $5 \% \mathrm{CO}_{2}$ at $37^{\circ} \mathrm{C}$. Wnt5a was purchased from R\&D Systems, Inc. (Minneapolis, MN, USA), and $1 \mathrm{mM}$ Wnt5a was used to treat AGS cells transfected with pcDNA3.1-ROR2 plasmid.

Reverse transcription-quantitative polymerase chain reaction (RT-qPCR). TRIzol ${ }^{\circledR}$ reagent (Thermo Fisher Scientific, Inc.) was used to extract total RNA from gastric carcinoma tissues or cell lines, according to the manufacturer's protocol. The concentration and quality of the RNA was analyzed using Nanodrop 2000 ultramicro spectrophotometer (Thermo Fisher Scientific, Inc.). Total RNA (500 ng) was reverse transcribed into cDNA using the RevertAid First Strand cDNA Synthesis kit (Thermo Fisher Scientific, Inc.), according to the manufacturer's protocol. qPCR was performed using the SYBR Green qPCR Assay kit (Toyobo Co., Ltd., Osaka, Japan), according to the manufacturer's protocol. The PCR cycling conditions were $50^{\circ} \mathrm{C}$ for $2 \mathrm{~min}$ and $95^{\circ} \mathrm{C}$ for $10 \mathrm{~min}$, followed by 40 cycles of denaturation at $95^{\circ} \mathrm{C}$ for $15 \mathrm{sec}$ and annealing/elongation at $60^{\circ} \mathrm{C}$ for $1 \mathrm{~min}$. The specific primer pairs were as follows: ROR2 sense, $5^{\prime}$-ATG GTTCACGACTGCGAATCC-3' and antisense, 5'-AATGGT CTTCATCCCGTTGGT-3'; and glyceraldehyde 3-phosphate dehydrogenase (GAPDH) sense, 5'-GGAGCGAGATCCCTC CAAAAT-3' and antisense, 5'-GGCTGTTGTCATACTTCT CATGG-3'. GAPDH was used as an internal control. cDNA was replaced with $\mathrm{H}_{2} \mathrm{O}$ for a negative control. Independent experiments were repeated three times. The relative mRNA expression levels of ROR2 were analyzed using the $2^{-\Delta \Delta C q}$ method (13).

Transfection. AGS cells were cultured to $70 \%$ confluence and resuspended in serum-free DMEM. Lipofectamine 2000 (Thermo Fisher Scientific, Inc.) was used to transfect the AGS cells with pcDNA3.1-ROR2 plasmid or blank pcDNA-3.1 vector as a negative control (NC). Briefly, serum-free medium was used to dilute the Lipofectamine 2000 or plasmid, after which the Lipofectamine 2000 and diluted plasmid were mixed. After incubation for $20 \mathrm{~min}$ at room temperature, the mixture was added to the AGS cell suspension and incubated at $37^{\circ} \mathrm{C}$ in $5 \%$ $\mathrm{CO}_{2}$ for $6 \mathrm{~h}$, followed by replacement of the medium with the normal serum-containing medium and incubation for $48 \mathrm{~h}$.

MTT assay. MTT assays were performed to determine the proliferation of AGS cells. Briefly, $10^{4}$ cells/well were seeded onto a 96-well plate and incubated for $6,12,24$ or $48 \mathrm{~h}$ at $37^{\circ} \mathrm{C}$ in $5 \% \mathrm{CO}_{2}$. Subsequently, $5 \mathrm{mg} / \mathrm{ml}$ MTT (Thermo Fisher Scientific, Inc.) was added to each well and the plates were incubated for a further $4 \mathrm{~h}$ at $37^{\circ} \mathrm{C}$ in $5 \% \mathrm{CO}_{2}$. The supernatant, obtained by centrifugation at $1,000 \times \mathrm{g}$ for $3 \mathrm{~min}$ at room temperature, was removed and $100 \mu \mathrm{l}$ dimethylsulfoxide (Thermo Fisher Scientific, Inc.) was added to dissolve the precipitate. The absorbance was detected at $492 \mathrm{~nm}$ using the Bio-Tek ${ }^{\mathrm{TM}}$ ELX-800 ${ }^{\mathrm{TM}}$ Absorbance Microplate reader (BioTek Instruments, Inc., Winooski, VT, USA).

Cell cycle distribution analysis. AGS cells $\left(5.0 \times 10^{6}\right.$ cells $\left./ \mathrm{ml}\right)$ were fixed in $70 \%$ ethanol overnight at $-20^{\circ} \mathrm{C}$, followed by two rounds of centrifugation at $1,000 \times \mathrm{g}$ for $5 \mathrm{~min}$. The cell pellets were then resuspended in $300 \mu \mathrm{l}$ propidium iodide (PI) staining buffer (Thermo Fisher Scientific, Inc.), and incubated for $30 \mathrm{~min}$ at room temperature. DNA content analyses were performed by flow cytometry.

Cell apoptosis assay. Cell apoptosis was determined using the Annexin V-fluorescein isothiocyanate (FITC) Apoptosis Detection kit (BD Pharmingen, San Diego, CA, USA). Briefly, AGS cells were harvested at $24 \mathrm{~h}$ post-transfection and washed twice with cold phosphate-buffered saline (PBS). Subsequently, $10^{6}$ cells were resuspended in $200 \mu \mathrm{l}$ binding buffer containing $10 \mu \mathrm{l}$ Annexin V-FITC and $5 \mu \mathrm{l}$ PI-phycoerythrin, and incubated in the dark for $30 \mathrm{~min}$ at $4^{\circ} \mathrm{C}$. Next, $300 \mu \mathrm{l}$ binding buffer was added to the cells and apoptosis was assessed by flow cytometry.

Western blotting. AGS cells were solubilized in cold radioimmunoprecipitation assay lysis buffer (Thermo Fisher Scientific, Inc.). Centrifugation was performed at $12,000 \mathrm{x} g$ for $30 \mathrm{~min}$ at $4^{\circ} \mathrm{C}$, and the supernatant was collected. Protein concentration was determined using a Pierce BCA Protein Assay kit (Thermo Fisher Scientific, Inc.), according to the manufacturer's instructions. Proteins $(50 \mu \mathrm{g})$ were separated by $10 \%$ SDS-PAGE (Pierce Biotechnology, Inc., Rockford, IL, USA), and transferred onto polyvinylidene difluoride membranes (Pierce Biotechnology, Inc.). The membranes were subsequently incubated with rabbit anti-ROR2 polyclonal antibody (1:50; cat. no. ab137602; Abcam, Cambridge, MA, USA), rabbit anti-c-Myc polyclonal antibody (1:100; cat. no. ab39688; Abcam), rabbit anti- $\beta$-catenin antibody (1:100; cat. no. ab32572) and rabbit anti-GAPDH polyclonal antibody (1:200; cat. no. ab9485; Abcam) for $3 \mathrm{~h}$ at room temperature. After washing three times with PBS containing Tween-20, the membranes were incubated with horseradish peroxidase-conjugated mouse anti-rabbit secondary antibody (1:10,000; cat. no. ab99697; Abcam) for $1 \mathrm{~h}$ at room temperature. Detection was performed using an Enhanced Chemiluminescence kit (Pierce Biotechnology, Inc.). The relative protein expression levels were determined using Image-Pro Plus 6.0 software (Media Cybernetics, Inc., Rockville, MD, USA), and are presented as the density ratio versus GAPDH.

Statistical analysis. Data are presented as the mean \pm standard deviation of at least three experiments. Statistical analysis was performed by one-way analysis of variance using SPSS 17.0 software (SPSS, Inc., Chicago, IL, USA). P<0.05 were considered to indicate a statistically significant difference.

\section{Results}

ROR2 is recurrently downregulated in gastric carcinoma tissues and cell lines. First, the present study examined 


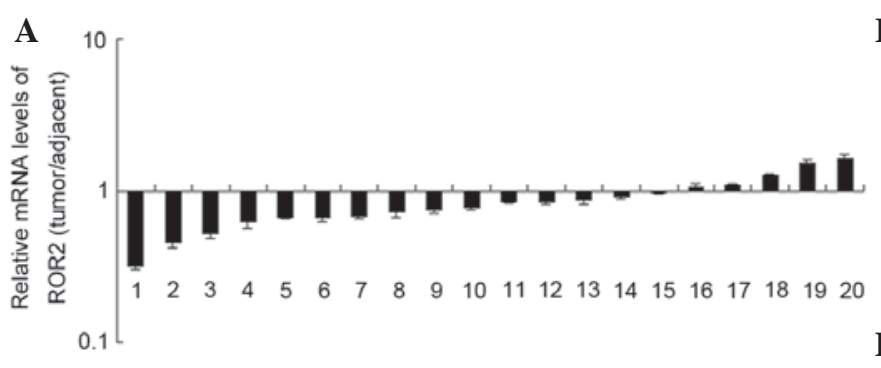

C

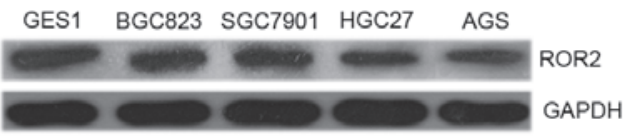

B

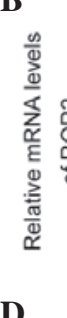

1.2

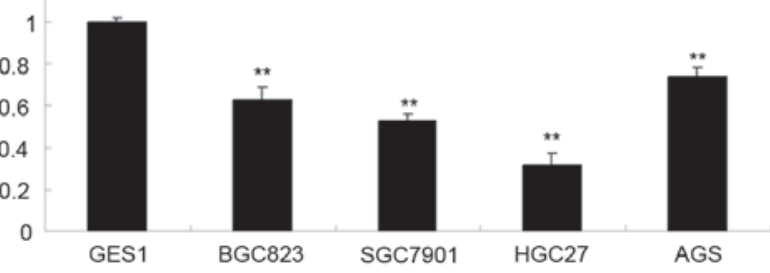

1.2

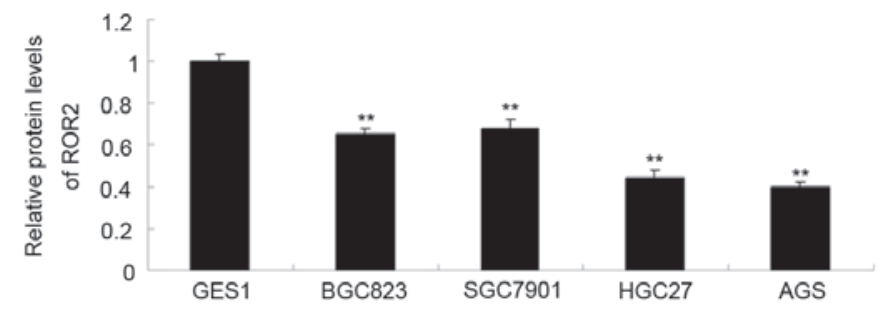

Figure 1. ROR2 expression was recurrently downregulated in gastric carcinoma tissues and cell lines. (A) The mRNA expression levels of ROR2 in gastric carcinoma tissues and their matched adjacent normal tissues were examined by RT-qPCR. The (B) mRNA and (C and D) protein expression levels of ROR2 in four common human gastric cancer cell lines (BGC823, SGC7901, HGC27 and AGS) and a normal gastric mucosa epithelial cell line (GES-1) were examined by RT-qPCR and western blotting, respectively. ${ }^{* *} \mathrm{P}<0.01$, vs. GES-1. ROR2, receptor tyrosine kinase-like orphan receptor 2; GAPDH, glyceraldehyde 3-phosphate dehydrogenase; RT-qPCR, reverse transcription-quantitative polymerase chain reaction.

the mRNA expression levels of ROR2 in gastric carcinoma tissues and cell lines using RT-qPCR. As is shown in Fig. 1A, the mRNA expression levels of ROR2 were recurrently downregulated in gastric carcinoma tissue samples, as compared with their matched adjacent normal tissue samples $(\mathrm{P}<0.01)$. As is shown in Fig. $1 \mathrm{~B}$ and $\mathrm{C}$, the mRNA and protein expression levels of ROR2 were significantly decreased in gastric carcinoma cell lines, as compared with GES1 cells $(\mathrm{P}<0.01)$. These results suggest that downregulation of ROR2 may be associated with the tumorigenesis of gastric carcinoma. Since the AGS gastric carcinoma cells exhibited the lowest ROR2 protein expression levels (Fig. 1C), AGS cells were selected for use in the subsequent experiments.

Overexpression of ROR2 inhibits the proliferation of AGS gastric carcinoma cells independently of Wnt5a. The ROR2 plasmid was transfected into gastric carcinoma AGS cells. As is shown in Fig. 2A and B, the relative mRNA and protein expression levels of ROR 2 were significantly increased following transfection, as compared with the control group $(\mathrm{P}<0.01)$, indicating that the transfection had been successful. The MTT assay was used to investigate the effect of ROR2 overexpression on AGS cell proliferation. As is shown in Fig. 2C, overexpression of ROR2 significantly inhibited the proliferation of AGS cells $(\mathrm{P}<0.01)$. However, addition of Wnt5a, the ligand of ROR2, did not affect the proliferation of ROR2-overexpressing AGS cells (P>0.05; Fig. 2D), thus suggesting that overexpression of ROR2 inhibits AGS cell proliferation independently of $\mathrm{Wnt} 5 \mathrm{a}$.

Overexpression of ROR 2 induces the apoptosis of AGS gastric carcinoma cells independent of Wnt5a. Cell apoptosis assays were performed to investigate the effect of ROR2 overexpression on AGS cell apoptosis. As is shown in Fig. 3A, the apoptotic level of AGS cells transfected with ROR2 plasmid was significantly increased, as compared with the control groups $(\mathrm{P}<0.01)$. Furthermore, the addition of Wnt5a did not affect the apoptosis level of ROR2-overexpressing AGS cells ( $\mathrm{P}>0.05$; Fig. 3B), thus suggesting that overexpression of ROR2 induces the apoptosis of AGS cells independently of Wnt5a.

Overexpression of ROR2 induces cell cycle arrest in AGS gastric carcinoma cells. Wnt signaling has previously been demonstrated to participate in the regulation of cell cycle progression (14). Therefore, the cell cycle distribution of AGS gastric carcinoma cells was examined for each group. As is shown in Fig. 4, ROR2-overexpressing AGS cells accumulated at the G1 phase, as compared with the control groups $(\mathrm{P}<0.01)$. These results suggest that overexpression of ROR2 induces an arrest in cell cycle progression at the G1 phase, and indicate that the induction of cell cycle arrest may be responsible for the reduced proliferation of ROR2-overexpressing AGS cells.

Overexpression of ROR2 suppresses the activation of canonical Wnt signaling. ROR 2 is a receptor of the non-canonical Wnt signaling pathway (15). Recently, ROR2 has been suggested to have an inhibitory effect on the activation of the canonical Wnt signaling pathway, which is involved in the regulation of cell survival and proliferation $(5,16)$. Since the present study demonstrated that overexpression of ROR2 inhibited AGS cell proliferation while inducing cell apoptosis and cell cycle arrest, it was hypothesized that overexpression of ROR2 may inhibit the activation of the canonical Wnt signaling pathway in AGS cells. To verify this, western blotting was performed to investigate the activation of the canonical Wnt signaling pathway, as well as c-Myc, which is an important downstream effecter responsible for the regulation of cell proliferation (17). As is shown in Fig. 5, overexpression of ROR2 significantly inhibited the protein expression levels of $\beta$-catenin in the nucleus, as well as the protein expression levels of c-Myc in AGS cells $(\mathrm{P}<0.01)$; thus suggesting that the activation of the canonical Wnt signaling 
A

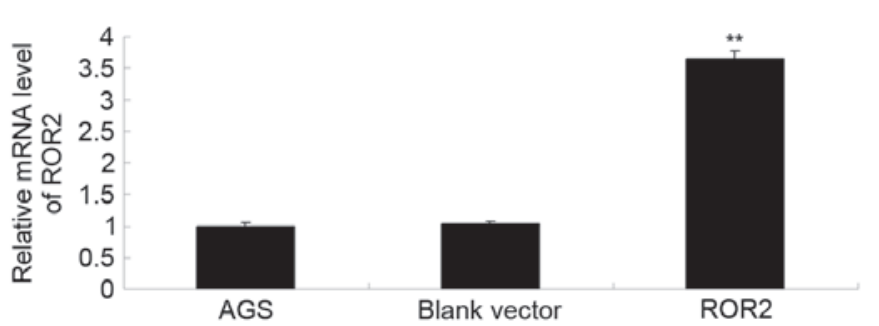

C

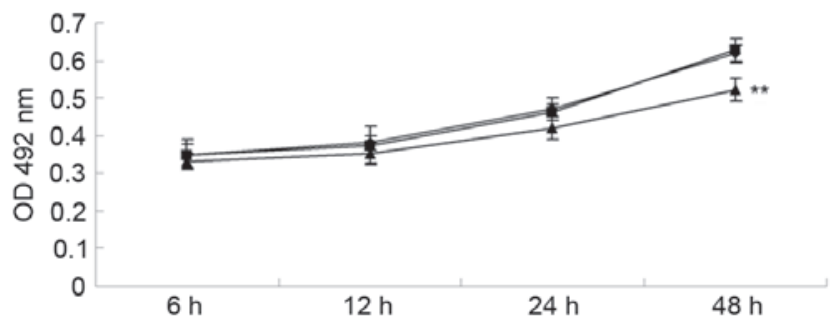

B
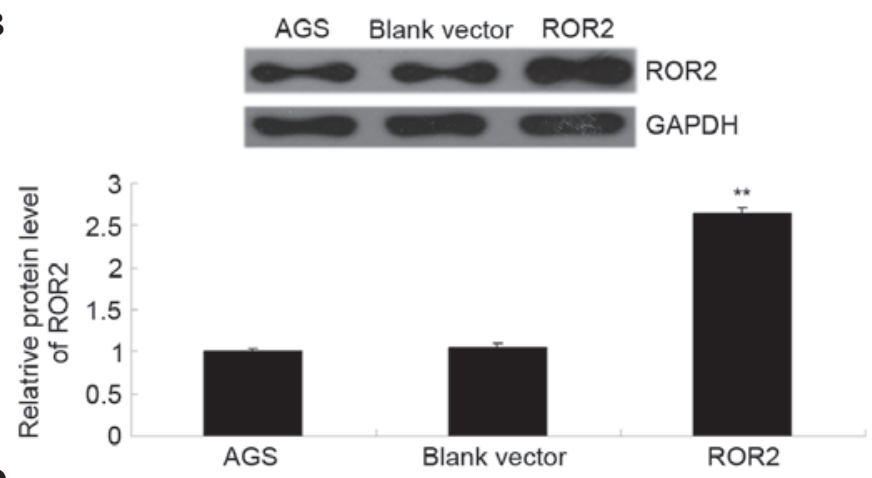

D

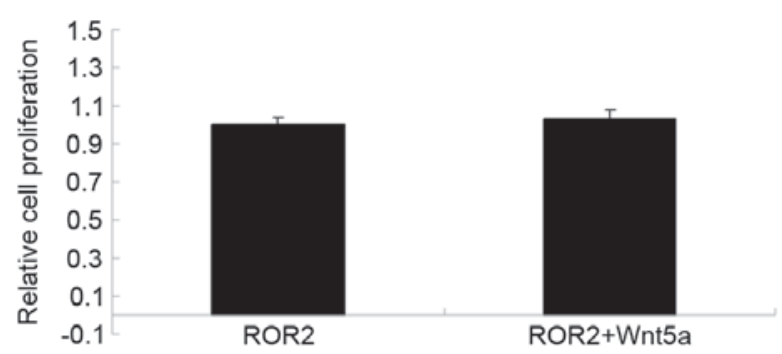

Figure 2. ROR2 overexpression inhibited the proliferation of AGS gastric carcinoma cells. The (A) mRNA and (B) protein expression levels of ROR2 in AGS cells transfected with the pcDNA3.1-ROR2 plasmid were examined by reverse transcription-quantitative polymerase chain reaction and western blotting, respectively. (C) MTT assays were performed to determine the proliferation capacity of AGS cells transfected with the pcDNA3.1-ROR2 plasmid. AGS cells transfected with the blank vector and non-transfected AGS cells were used as controls. (D) MTT assays were performed to determine the proliferation capacity of AGS cells transfected with the pcDNA3.1-ROR2 plasmid in the presence or absence of the ROR2 ligand, Wnt5a. ${ }^{* *} \mathrm{P}<0.01$, vs. AGS. ROR2, receptor tyrosine kinase-like orphan receptor 2; GAPDH, glyceraldehyde 3-phosphate dehydrogenase; OD, optical density.

pathway was inhibited in ROR-overexpressing cells. Theses results suggest that the inhibitory effect of ROR 2 overexpression on AGS cell proliferation may be due to the suppression of canonical Wnt signaling.

\section{Discussion}

ROR2 is a type I transmembrane protein that belongs to the ROR subfamily of cell surface receptors (18). Previous studies have suggested that the deregulation of ROR2 is a key factor during tumorigenesis, and that ROR2 is involved in the development of some common types of human cancers $(5,11,12)$. For instance, the expression levels of ROR2 were associated with the expression of genes involved in the extracellular matrix, including Twist and matrix metalloproteinase-2, in RCC (12). In addition, knockdown of ROR2 inhibited RCC cell migration, as well as anchorage-independent growth in soft agar and growth in an orthotopic xenograft model (12); thus suggesting that ROR2 has an oncogenic role in RCC. Furthermore, ROR2 was reported to promote the development of various other types of cancer, including melanoma and oral squamous cell carcinoma $(15,19)$.

Conversely, ROR2 has been reported to exert a suppressive role in other types of human cancers. Li et al (10) demonstrated that ROR2 was recurrently silenced by $\mathrm{CpG}$ methylation at its promoter in nasopharyngeal, esophageal, colorectal, gastric, hepatocellular, lung and breast cancer cell lines. The present study demonstrated that the expression levels of ROR2 were recurrently reduced in gastric carcinoma tissues, as compared with their matched normal adjacent tissues. In addition, ROR 2 expression was significantly decreased in several common gastric carcinoma cell lines, as compared with normal gastric epithelial cells. These results suggested that the aberrant downregulation of ROR2 may be associated with the development and progression of gastric carcinoma.

In the present study, AGS gastric carcinoma cells were transfected with an ROR2 plasmid, and the expression levels of ROR2 were observed to have been successfully increased following transfection. Overexpression of ROR2 markedly inhibited AGS cell proliferation, while inducing cell apoptosis and cell cycle arrest at the G1 phase. Similarly, Li et al (10) reported that ectopic expression of ROR2 inhibited tumor cell growth and induced cell cycle arrest and apoptosis. Furthermore, ROR2 was revealed to suppress epithelial-mesenchymal transition, migration and invasion (10).

Lu et al (11) demonstrated that the expression levels of ROR2 and Wnt5a were significantly upregulated in osteosarcoma, and that their expression levels were associated with the malignant progression. Therefore, the present study investigated the effect of Wnt5a on the proliferation and apoptosis of gastric carcinoma cells. However, it was demonstrated that the addition of Wnt5a did not affect the proliferation or the apoptosis of AGS cells transfected with an ROR2 plasmid; thus suggesting that the inhibitory effects of ROR2 overexpression on gastric carcinoma may be independent of Wnt5a.

The canonical Wnt signaling pathway has been observed to be involved in embryonic development, cell differentiation and tumorigenesis (20-22). In addition, aberrant activation of the canonical Wnt signaling pathway has been demonstrated in a variety of human malignancies, including gastric carcinoma (23-27). Therefore, inhibition of canonical Wnt signaling may be a promising strategy for the treatment of 
A
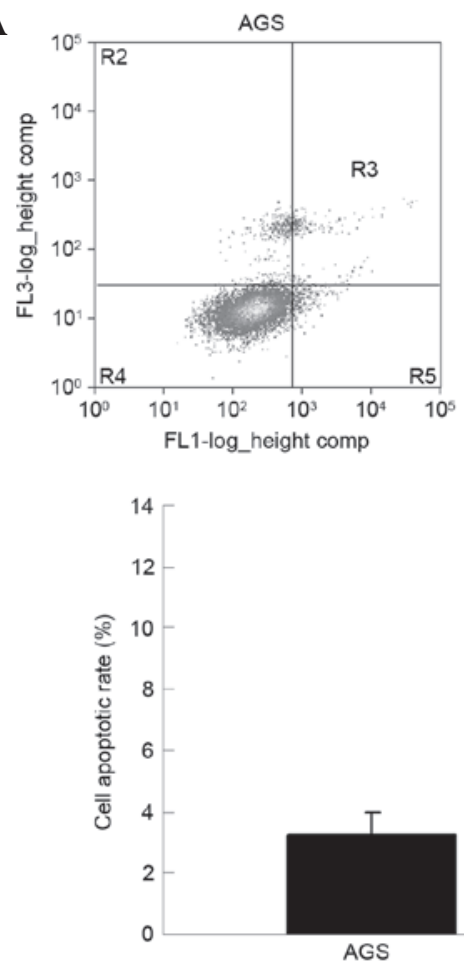
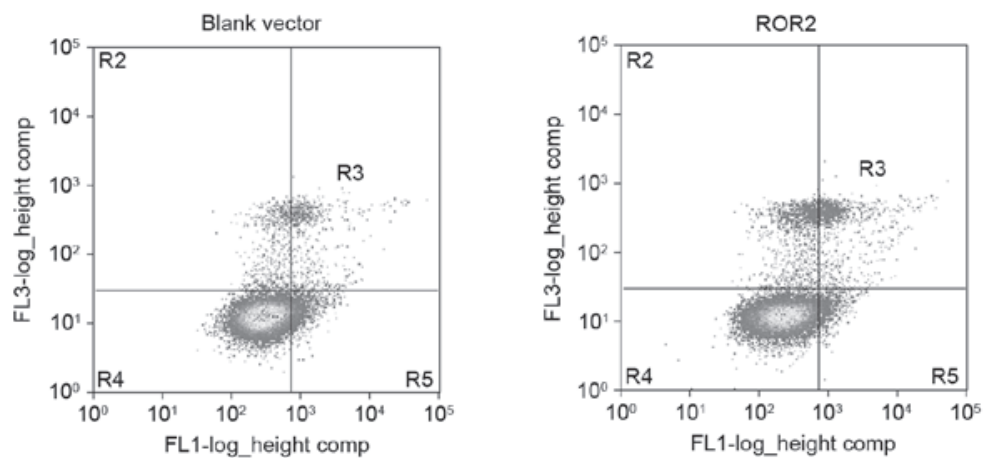

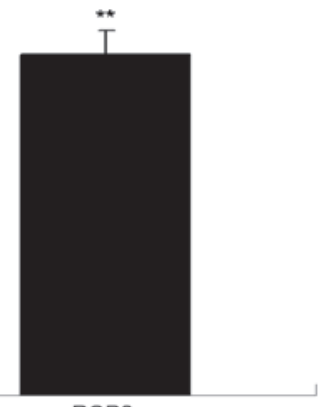

Blank vector

ROR2

B
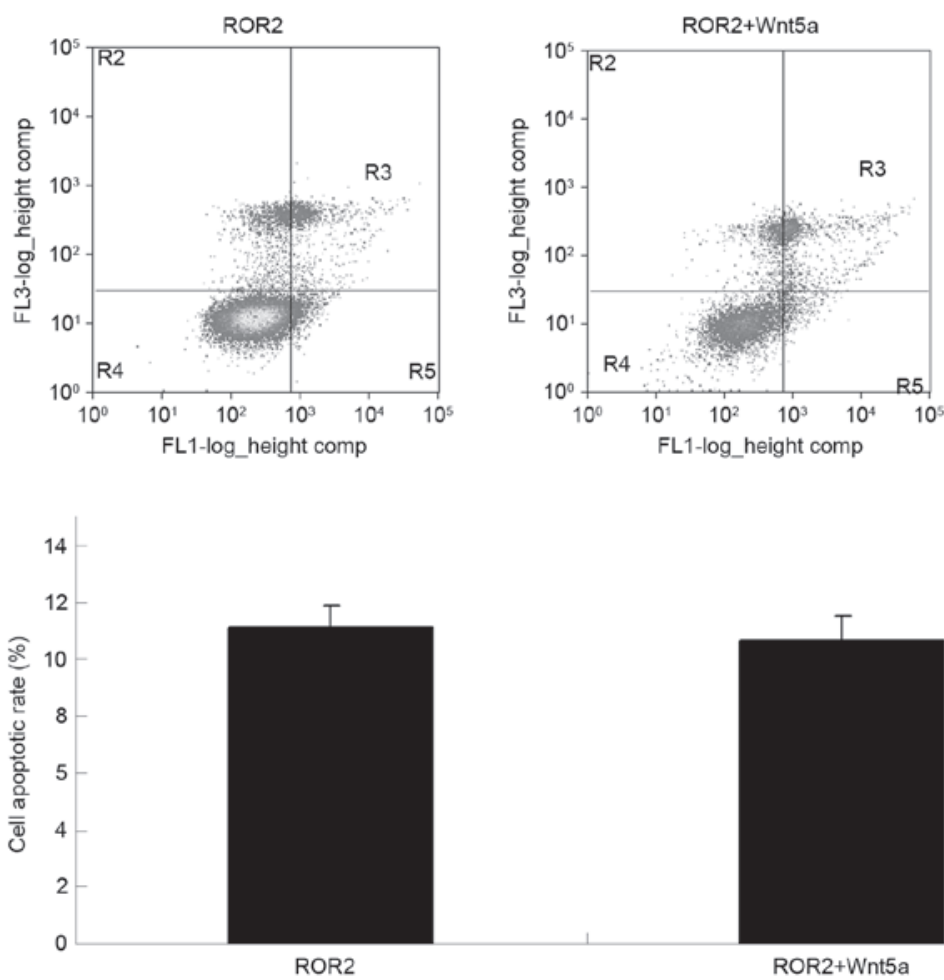

ROR2+Wnt5a

Figure 3. ROR2 overexpression induced the apoptosis of AGS gastric cancer cells. (A) Cell apoptosis assays were conducted for AGS cells transfected with the pcDNA3.1-ROR2 plasmid. AGS cells transfected with the blank vector and non-transfected AGS cells were used as controls. (B) Cell apoptosis assays were conducted for AGS cells transfected with the pcDNA3.1-ROR2 plasmid in the presence or absence of the ROR2 ligand, Wnt5a. ${ }^{* *} \mathrm{P}<0.01$, vs. AGS. ROR2, receptor tyrosine kinase-like orphan receptor 2.

gastric carcinoma. The present study investigated whether overexpression of the non-canonical Wnt receptor ROR2 was able to alter the activation of the canonical Wnt signaling pathway. It was demonstrated that overexpression of ROR2 significantly decreased the protein expression level of $\beta$-catenin in the nucleus of AGS cells, as well as the protein expression levels of c-Myc, an important downstream effector in the canonical Wnt signaling pathway (17). These results 

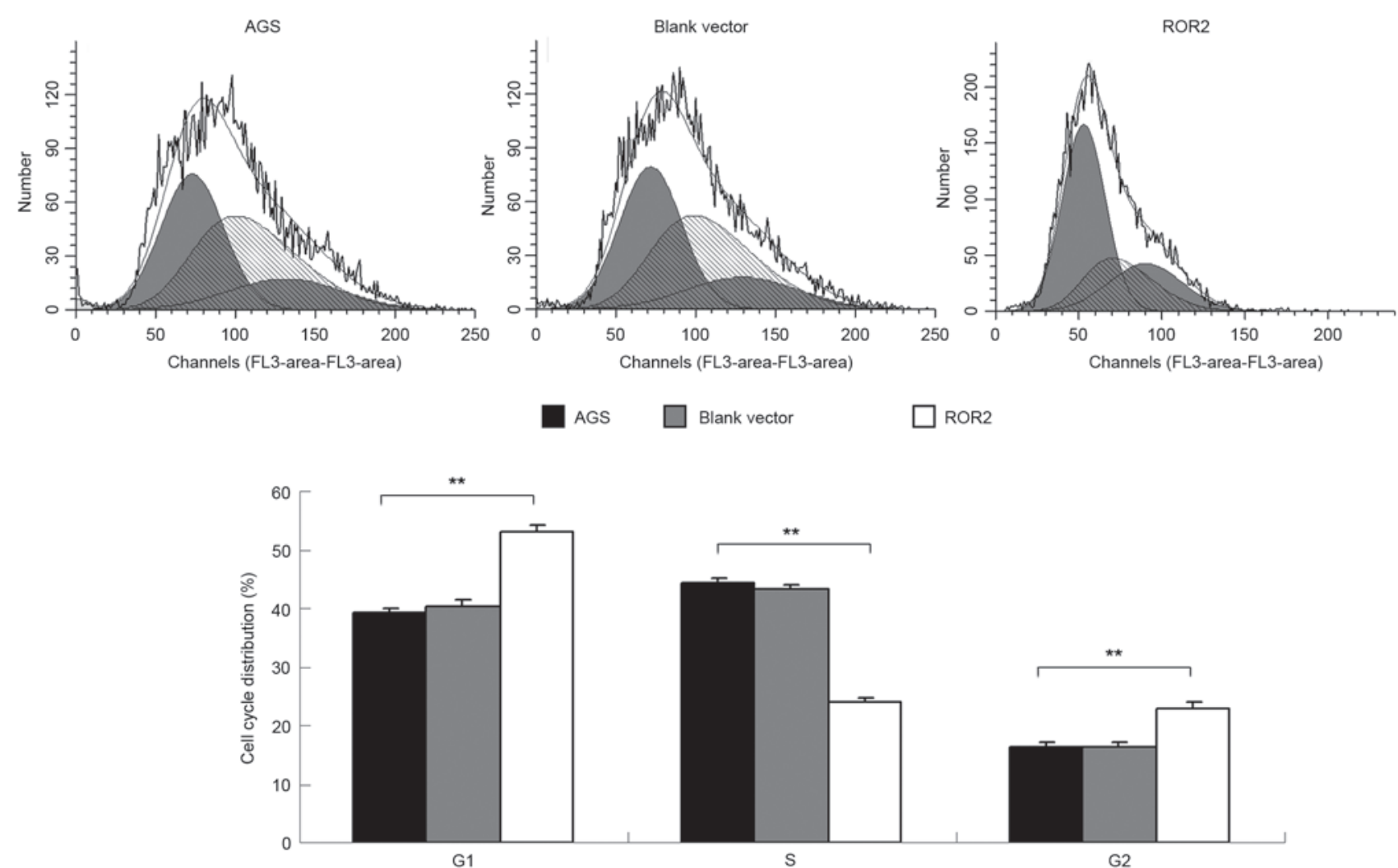

Figure 4. ROR2 overexpressing AGS cells accumulated at the G1 phase. Cell cycle distribution assays were conducted for AGS cells transfected with the pcDNA3.1-ROR2 plasmid. AGS cells transfected with the blank vector and non-transfected AGS cells were used as controls. ${ }^{* *} \mathrm{P}<0.01$, vs. AGS. ROR2, receptor tyrosine kinase-like orphan receptor 2.

A
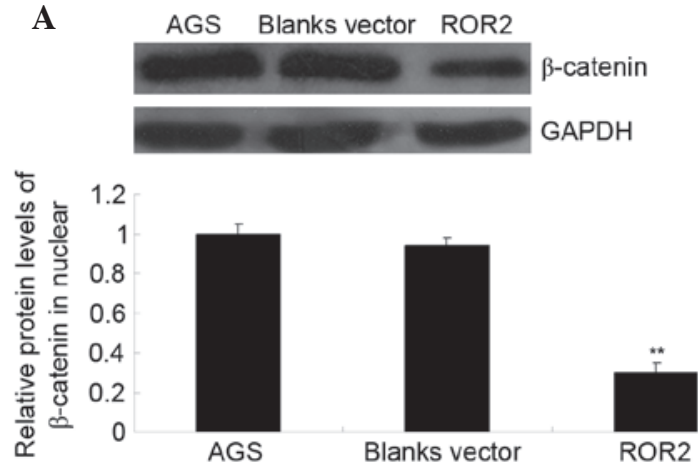

$\mathbf{B}$
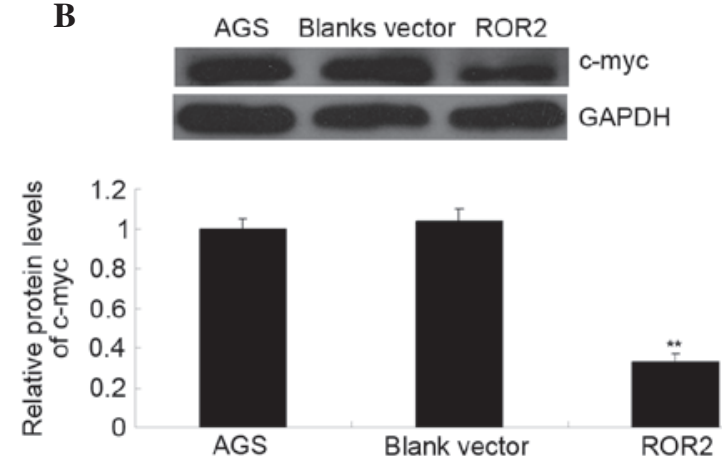

Figure 5. ROR2 overexpression suppresses canonical Wnt signaling. Western blotting was performed to examine the protein expression levels of (A) $\beta$-catenin in the nucleus and (B) c-Myc in AGS cells transfected with the pcDNA3.1-ROR2 plasmid. GAPDH was used as an internal reference. AGS cells transfected with the blank vector and non-transfected AGS cells were used as controls. ${ }^{* *} \mathrm{P}<0.01$, vs. AGS. ROR2, receptor tyrosine kinase-like orphan receptor 2; GAPDH, glyceraldehyde 3-phosphate dehydrogenase. suggested that activation of non-canonical Wnt signaling may inhibit the activity of the canonical Wnt signaling pathway in AGS gastric carcinoma cells.

In addition to gastric carcinoma, ROR 2 expression levels were reported to be significantly decreased in hepatocellular carcinoma (HCC) tissues, and this was associated with a poor prognosis (28); thus suggesting that ROR2 may act as a tumor suppressor in HCC. Therefore, ROR2 appears to exert dual roles as an oncogene or tumor suppressor depending on the tumor type (5).

The main limitation of the present study was that the sample volume of gastric cancer tissues was not sufficient to examine the association between the expression levels of ROR 2 and Wnt5a, and the clinical characteristics of patients with gastric cancer, such as tumor grade, TNM stage and metastasis. The results of the present study suggested that ROR2 induced the apoptosis of AGS cells independently of Wnt5a. Therefore, future studies are required to elucidate the underlying mechanism by which ROR 2 affects gastric cancer cell apoptosis.

In conclusion, the present study demonstrated that ROR2 was recurrently downregulated in gastric carcinoma tissues and cell lines. Furthermore, overexpression of ROR2 significantly inhibited the proliferation and induced the apoptosis and cell cycle arrest of gastric carcinoma cells. An investigation of the underlying molecular mechanism demonstrated that ROR2 overexpression inhibited the activity of the canonical Wnt signaling pathway. The results of the present study suggested that ROR2 may emerge as a potential candidate for the treatment of gastric carcinoma. 


\section{References}

1. Shi J, Qu YP and Hou P: Pathogenetic mechanisms in gastric cancer. World J Gastroenterol 20: 13804-13819, 2014.

2. Piazuelo MB and Correa P: Gastric cancer: Overview. Colomb Med (Cali) 44: 192-201, 2013.

3. Li Z, Lei H, Luo M, Wang Y, Dong L, Ma Y, Liu C, Song W, Wang F, Zhang J, et al: DNA methylation downregulated mir-10b acts as a tumor suppressor in gastric cancer. Gastric Cancer 18: 43-54, 2014.

4. Qiu T, Zhou X, Wang J, Du Y, Xu J, Huang Z, Zhu W, Shu Y and Liu P: MiR-145, miR-133a and miR-133b inhibit proliferation, migration, invasion and cell cycle progression via targeting transcription factor Sp1 in gastric cancer. FEBS Lett 588: 1168-1177, 2014.

5. Ford CE, Qian Ma SS, Quadir A and Ward RL: The dual role of the novel Wnt receptor tyrosine kinase, ROR2, in human carcinogenesis. Int J Cancer 133: 779-787, 2013.

6. Sundaram MV: Canonical RTK-Ras-ERK signaling and related alternative pathways. WormBook: 1-38, 2013

7. Jimenez G, Shvartsman SY and Paroush Z: The Capicua repressor-a general sensor of RTK signaling in development and disease. J Cell Sci 125: 1383-1391, 2012.

8. Batchu SN and Korshunov VA: Novel tyrosine kinase signaling pathways: Implications in vascular remodeling. Curr Opin Nephrol Hypertens 21: 122-127, 2012.

9. Enomoto M, Hayakawa S, Itsukushima S, Ren DY, Matsuo M, Tamada K, Oneyama C, Okada M, Takumi T, Nishita M and Minami Y: Autonomous regulation of osteosarcoma cell invasiveness by Wnt5a/Ror2 signaling. Oncogene 28: 3197-3208, 2009.

10. Li L, Ying J, Tong X, Zhong L, Su X, Xiang T, Shu X, Rong R, Xiong L, Li H, et al: Epigenetic identification of receptor tyrosine kinase-like orphan receptor 2 as a functional tumor suppressor inhibiting $\beta$-catenin and AKT signaling but frequently methylated in common carcinomas. Cell Mol Life Sci 71: 2179-2192, 2014.

11. Lu BJ, Wang YQ, Wei XJ, Rong LQ, Wei D, Yan CM, Wang DJ and Sun JY: Expression of WNT-5a and ROR2 correlates with disease severity in osteosarcoma. Mol Med Rep 5: 1033-1036, 2012.

12. Wright TM, Brannon AR, Gordan JD, Mikels AJ, Mitchell C Chen S, Espinosa I, van de Rijn M, Pruthi R, Wallen E, et al: Ror2, a developmentally regulated kinase, promotes tumor growth potential in renal cell carcinoma. Oncogene 28: 2513-2523, 2009.

13. Livak KJ and Schmittgen TD: Analysis of relative gene expression data using real-time quantitative PCR and the 2(-Delta Delta C(T)) Method. Methods 25: 402-408, 2001.

14. Katoh M: WNT signaling pathway and stem cell signaling network. Clin Cancer Res 13: 4042-4045, 2007.

15. O'Connell MP, Fiori JL, Xu M, Carter AD, Frank BP, Camilli TC, French AD, Dissanayake SK, Indig FE, Bernier M, et al: The orphan tyrosine kinase receptor, ROR2, mediates Wnt5A signaling in metastatic melanoma. Oncogene 29: 34-44, 2010.
16. Green J, Nusse R and van Amerongen R: The role of Ryk and Ror receptor tyrosine kinases in Wnt signal transduction. Cold Spring Harb Perspect Biol 6: 2014.

17. Heo SH, Jeong ES, Lee KS, Seo JH, Jeong DG, Won YS, Kwon HJ, Kim HC, Kim DY and Choi YK: Canonical Wnt signaling pathway plays an essential role in N-methyl-N-nitrosurea induced gastric tumorigenesis of mice. J Vet Med Sci 75: 299-307, 2013.

18. Stricker S and Mundlos S: FGF and ROR2 receptor tyrosine kinase signaling in human skeletal development. Curr Top Dev Biol 97: 179-206, 2011.

19. Kobayashi M, Shibuya Y, Takeuchi J, Murata M, Suzuki H, Yokoo S, Umeda M, Minami Y and Komori T: Ror2 expression in squamous cell carcinoma and epithelial dysplasia of the oral cavity. Oral Surg Oral Med Oral Pathol Oral Radiol Endod 107: 398-406, 2009.

20. Lerner UH and Ohlsson C: The WNT system: Background and its role in bone. J Intern Med 277: 630-649, 2015.

21. Jones AE, Price FD, Le Grand F, Soleimani VD, Dick SA, Megeney LA and Rudnicki MA: Wnt/ $\beta$-catenin controls follistatin signalling to regulate satellite cell myogenic potential. Skelet Muscle 5: 14, 2015.

22. He H, Chen K, Wang F, Zhao L, Wan X, Wang L and Mo Z miR204-5p promotes the adipogenic differentiation of human adipose-derived mesenchymal stem cells by modulating DVL3 expression and suppressing Wnt/beta-catenin signaling. Int J Mol Med 35: 1587-1595, 2015.

23. Chang B, Tessneer KL, McManus J, Liu X, Hahn S, Pasula S, Wu H, Song H, Chen Y, Cai X, et al: Epsin is required for dishevelled stability and Wnt signalling activation in colon cancer development. Nat Commun 6: 6380, 2015.

24. Xie J, Zhang Y, Hu X, Lv R, Xiao D, Jiang L and Bao Q: Norcantharidin inhibits Wnt signal pathway via promoter demethylation of WIF-1 in human non-small cell lung cancer. Med Oncol 32: 145,2015

25. Ashihara E, Takada T and Maekawa T: Targeting the canonical Wnt $/ \beta$-catenin pathway in hematological malignancies. Cancer Sci 106: 665-671, 2015.

26. Ilmer M, Boiles AR, Regel I, Yokoi K, Michalski CW, Wistuba II, Rodriguez J, Alt E and Vykoukal J: RSPO2 enhances canonical wnt signaling to confer stemness-associated traits to susceptible pancreatic cancer cells. Cancer Res 75: 1883-1896, 2015.

27. Fang F, Zhao WY, Li RK, Yang XM, Li J, Ao JP, Jiang SH, Kong FZ, Tu L, Zhuang C, et al: Silencing of WISP3 suppresses gastric cancer cell proliferation and metastasis and inhibits Wnt/ $\beta$-catenin signaling. Int J Clin Exp Pathol 7: 6447-6461, 2014.

28. Geng M, Cao YC, Chen YJ, Jiang H, Bi LQ and Liu XH: Loss of Wnt5a and Ror2 protein in hepatocellular carcinoma associated with poor prognosis. World J Gastroenterol 18: 1328-1338, 2012. 